\title{
Efficacy And Safety Of Raltitrexed Plus Oxaliplatin-Based Transarterial Chemoembolization In Patients With Unresectable Hepatocellular Carcinoma
}

This article was published in the following Dove Press journal:

Cancer Management and Research

\begin{abstract}
Wenbo Shao, ',* Caixia $\mathrm{Li}^{2, *}$ Jun Tang, ${ }^{3}$ Jinlong Song,' Zixiang $\mathrm{Li}^{4},{ }^{4}$ Jize Sun, ${ }^{5}$ Yili $\mathrm{Xu}{ }^{6}$ Zhaomin Zheng, ${ }^{7}$ Jingqin Cao, Lei Zhang ${ }^{3}$

'Department of Surgical Oncology (Interventional Therapy), Shandong Cancer Hospital and Institute, Shandong First Medical University and Shandong Academy of Medical Sciences, Jinan, People's Republic of China; ${ }^{2}$ Department of Interventional Radiology, Qilu Hospital of Shandong University, Jinan, People's Republic of China; ${ }^{3}$ Department of Interventional Radiology, Shandong Medical Imaging Research Institute, Jinan, People's Republic of China; ${ }^{4}$ Department of Interventional Radiology, Affiliated Hospital of Qingdao University, Qingdao, People's Republic of China; ${ }^{5}$ Department of Interventional Radiology, Qingdao Central Hospital, Qingdao, People's Republic of China; ${ }^{6}$ Department of Interventional Radiology, 960 Hospital of People's Liberation Army, Tai'an, People's Republic of China; 'Department of Interventional Radiology, Qianfoshan Hospital of Shandong Province, Jinan, People's Republic of China; ${ }^{8}$ Department of Interventional Radiology, People's Hospital of Jining City, Jining, People's Republic of China

*These authors contributed equally to this work
\end{abstract}

Correspondence: Jun Tang

Department of Interventional Radiology, Shandong Medical Imaging Research Institute, 324 Jingwu Road, Jinan, Shandong 25002 I, People's Republic of

China

Tel +86 I3964031909

Email tangjun7925032@I63.com

Jinlong Song

Department of Surgical Oncology (Interventional Therapy), Shandong

Cancer Hospital and Institute, Shandong First Medical University and Shandong

Academy of Medical Sciences, 440 Jiyan

Road, Jinan, Shandong 250I I7, People's

Republic of China

Tel +86 I3969080678

Email jls7286@I63.com
Objective: To evaluate the efficacy and safety of raltitrexed plus oxaliplatin-based transarterial chemoembolization (TACE) in patients with unresectable hepatocellular carcinoma (HCC).

Methods: A total of 123 patients with unresectable HCC were recruited into the prospective cohort study. Raltitrexed plus oxaliplatin-based TACE was performed according to the traditional method at monthly intervals and was repeated for up to 4 cycles if no disease progression or intolerable toxicity occurred. The primary efficacy endpoint was overall survival (OS), and the secondary endpoints were progression-free survival (PFS) and tumor response rate. The Cox proportional-hazards regression model was used to assess the independent prognostic factors of OS. Adverse events were also observed.

Results: The median OS time and PFS were 623 days (95\% CI: 461, 785) and 338 days (95\% CI: 302, 704), respectively. The disease control rate was 95.5\% (118/123). The Cox proportional-hazards regression model indicated that age, ECOG performance status and response to TACE as independent prognostic factors of OS. No treatment-related mortality occurred within 30 days of treatment procedure. The most common complications included postembolization syndrome, liver dysfunction and hematological toxicity. Grade 3 pain, transglutaminase abnormality and thrombocytopenia were observed in $16(13 \%), 15$ $(12.2 \%)$ and $3(2.4 \%)$ patients, respectively. No grade 4 adverse events were observed.

Conclusion: Raltitrexed plus oxaliplatin-based TACE led to high tumor response rate and promising PFS and OS, and was considered safe and tolerable in patients with unresectable HCC. Keywords: raltitrexed, oxaliplatin, hepatocellular carcinoma, transarterial chemoembolization

\section{Introduction}

Transarterial chemoembolization (TACE) is most commonly recommended as the first-line treatment for unresectable hepatocellular carcinoma (HCC) ${ }^{1,2}$ and also as the standard treatment for intermediate-stage HCC. ${ }^{3}$ However, no uniform standard protocol for TACE has been adopted globally. ${ }^{2}$ The choice of embolizing agents used was differed by different centers, and also the schedule and interval of retreatment, especially the choice of anticancer chemotherapeutic agents used. Several chemotherapeutic agents, including doxorubicin, cisplatin, fluorouracil and mitomycin, have been used along with TACE either as a sole agent or as a combination drug regimen. ${ }^{4}$ There is no high-quality evidence for choosing the best 
chemotherapeutic agent currently. It is imperative to explore the efficacy and safety of a new agent or regimen for treating HCC.

According to a randomized, multicenter, open-label study conducted in 2013, FOLFOX4 (infusion 5-fluorouracil, leucovorin and oxaliplatin) chemotherapy demonstrated better progression-free survival (PFS) and response rates when compared with doxorubicin alone in patients with advanced HCC. ${ }^{5}$ Many studies have shown that intra-arterial perfusion provides higher response rates than conventional systemic chemotherapy, ${ }^{6-8}$ and it is natural to the hypothesis that transarterial infusion of FOLFOX4 might be more effective than systemic chemotherapy. However, transarterial infusion of FOLFOX 4 might be problematic as 5-fluorouracil, a component of FOLFOX4, is a time-dependent antitumor agent and requires prolonged infusion for approximately 44-48 hrs. ${ }^{6}$ This increases the risk of catheter thrombosis and limits the mobility of patients when the catheter is inserted from the common femoral artery.

Raltitrexed is another thymidylate synthase inhibitor that is administered as a short-term infusion or mixed with lipiodol, and so it is regarded as a better candidate for TACE when compared with 5-fluorouracil. Many studies have shown that raltitrexed-based chemotherapy regimen has similar efficacy to that of FOLFOX4 in patients with advanced colorectal cancer, ${ }^{9,10}$ and revealed a safe and effective hepatic arterial infusion of raltitrexed and oxaliplatin in patients with colorectal cancer. ${ }^{6,10-13}$ However, the clinical data of raltitrexed in TACE for HCC are rare when compared with the data of hepatic arterial infusion of raltitrexed for advanced colorectal cancer. Hence, in the present study, the efficacy and safety of raltitrexed plus oxaliplatin-based TACE was evaluated in patients with unresectable HCC.

\section{Patients And Methods Selection Of Patients}

This multicenter, prospective cohort study was conducted in accordance with the Declaration of Helsinki. This study was approved by the Institutional Ethics Committee of Shandong Medical Imaging Research Institute and obtained approval from the ethics committee of Shandong Cancer Hospital and Institute, Qilu Hospital of Shandong University, Affiliated Hospital of Qingdao University, Qingdao Central Hospital, 960 Hospital of People's Liberation Army, Qianfoshan Hospital of Shandong Province and People's Hospital of Jining City. All patients provided written informed consent form. Patients were recruited from 8 centers of Shandong
Table I Baseline Characteristics Of The Study Population

\begin{tabular}{|c|c|c|}
\hline Age & $58(38-77)$ & $\mathbf{N}(\%)$ \\
\hline \multirow[t]{2}{*}{ Age } & $<60$ & $82(66.7 \%)$ \\
\hline & $\geq 60$ & $4 \mathrm{I}(33.3 \%)$ \\
\hline \multirow[t]{2}{*}{ Gender } & Male & $100(81.3 \%)$ \\
\hline & Female & $23(18.7 \%)$ \\
\hline \multirow[t]{3}{*}{ ECOG } & 0 & $63(51.2 \%)$ \\
\hline & I & $50(40.1 \%)$ \\
\hline & 2 & $10(8.1 \%)$ \\
\hline \multirow[t]{3}{*}{ Etiology } & Hepatitis B virus & 87 (70.7\%) \\
\hline & Hepatitis C virus & $2(1.6 \%)$ \\
\hline & Cirrhosis & $12(9.8 \%)$ \\
\hline \multirow[t]{3}{*}{ Location of tumor } & Right lobe & 77 (62.6\%) \\
\hline & Left lobe & 27 (22.0\%) \\
\hline & Bilobar & 19 (I5.4\%) \\
\hline \multirow[t]{2}{*}{ Number of tumors } & $\mathrm{I}-3$ & 99 (80.4\%) \\
\hline & $>3$ & 24 (19.5\%) \\
\hline \multirow[t]{2}{*}{ Lengths of the tumors } & $\leq 10 \mathrm{~cm}$ & 89 (72.4\%) \\
\hline & $>10 \mathrm{~cm}$ & $34(27.6 \%)$ \\
\hline \multirow[t]{2}{*}{ Distant metastasis } & Yes & $69(56.1 \%)$ \\
\hline & No & 54 (43.9\%) \\
\hline \multirow[t]{2}{*}{ AFP } & $\leq 200 \mathrm{ng} / \mathrm{mL}$ & $69(56.1 \%)$ \\
\hline & $>200 \mathrm{ng} / \mathrm{mL}$ & 54 (43.9\%) \\
\hline \multirow[t]{2}{*}{ Child-Pugh Score } & $A$ & $84(68.3 \%)$ \\
\hline & B & 39 (31.7\%) \\
\hline
\end{tabular}

Province in China. The inclusion criteria were as follows: (1) patients aged $>18$ years with $\mathrm{HCC}$ who are unsuitable for resection or percutaneous ablation; (2) Eastern Cooperative Oncology Group (ECOG) performance status of less than or equal to 2; (3) preserved liver function (Child-Pugh Class A or B); (4) a life expectancy of more than 12 weeks and (5) a leukocyte count of $>4.0 \times 10^{9} / \mathrm{L}$, platelet count $\geq 80 \times 10^{9} / \mathrm{L}$, hemoglobin $(\mathrm{Hb}) \geq 80 \mathrm{~g} / \mathrm{L}$; creatinine $(\mathrm{Cr}) \leq 2.0 \times \mathrm{UNL}$ (upper normal limits), bilirubin (BIL) $\leq 2.0 \times \mathrm{UNL}$, alanine transaminase (ALT) and aspartate transaminase (AST) $\leq 5.0 \times \mathrm{UNL}$. All patients were excluded if they had any other primary tumors, severe infection, known central nervous system metastases, and other serious illnesses or medical conditions.

\section{Treatment Plan}

TACE was performed according to the traditional method. The Seldinger technique was used to obtain access to the femoral artery. Arteriography of the hepatic artery was performed to localize the tumor and obtain information regarding the feeding arteries. The tumor-feeding artery was 


\section{Survival Function}

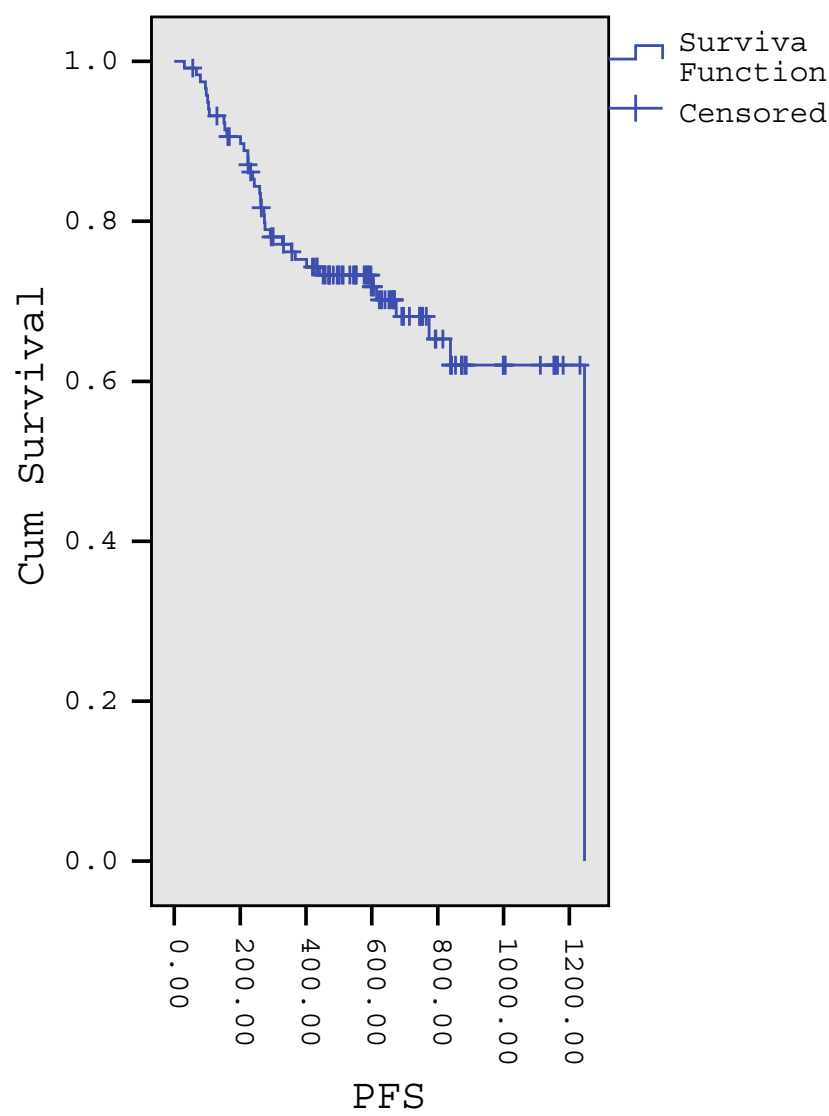

Figure I Kaplan - Meier curve showing the median progression-free survival in patients with unresectable hepatocellular carcinoma treated using raltitrexed plus oxaliplatin-based TACE.

selected or super-selected prior to chemoembolization. Oxaliplatin (100-200 mg) was infused into the feeding artery of the tumor, followed by infusion of $5-20 \mathrm{~mL}$ of lipiodol mixed with raltitrexed (2-4 mg) into the feeding artery until stasis flow in tumor vascularity was achieved. Gelatin sponge particles were used after embolization with lipiodol emulsion, but the use of gelatin sponge was not obliged and determined by the interventional radiologist. TACE was performed at monthly intervals and repeated for up to 4 cycles if no disease progression or intolerable toxicity occurred.

\section{Outcomes Assessment \\ Efficacy}

The primary efficacy endpoint was overall survival (OS), and the secondary endpoints were PFS and tumor response rate. Tumor responses were assessed using the modified response evaluation criteria in solid tumors (mRECIST). Contrastenhanced computed tomography (CT) or magnetic resonance

\section{Survival Function}

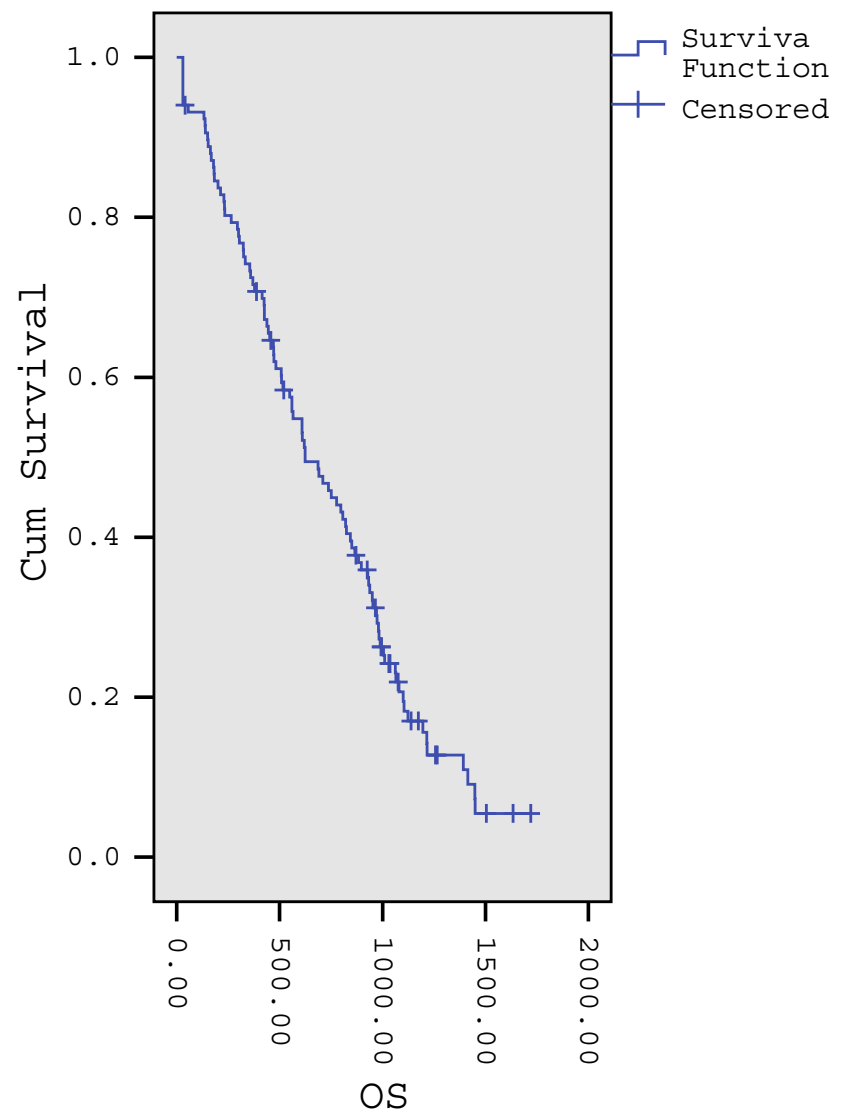

Figure 2 Kaplan - Meier curve showing the median overall survival in patients with unresectable hepatocellular carcinoma treated using raltitrexed plus oxaliplatinbased TACE.

imaging (MRI) and laboratory analyses including complete blood cell count, blood chemistry and tumor marker level were carried out within 7 days of initiating TACE. Repeated CT/MRI and laboratory analyses were performed after 4 weeks of every TACE cycle. TACE was repeated for up to 4 cycles if no complete responses or disease progression occurred. After 4 cycles of TACE, patients were subjected to follow-up every 12 weeks for assessing PFS or OS until disease progression or death.

\section{Safety}

The primary safety endpoint was the incidence of treatmentrelated serious adverse events (SAEs) that occur within 30 days of treatment procedure. Secondary safety outcomes included the incidence and severity of adverse events (AEs) that were assessed according to the National Cancer Institute Common Toxicity criteria (Version 3.0) after every TACE 
Table 2 Cox Regression Of Independent Prognostic Factor For Overall Survival

\begin{tabular}{|c|c|c|c|}
\hline & \multicolumn{2}{|c|}{ Cox Regression } & \multirow[t]{2}{*}{$\mathbf{P}$} \\
\hline & HR & $95 \% \mathrm{Cl}$ & \\
\hline Age $(\leq 60,>60)$ & 0.443 & $0.112-2.262$ & 0.043 \\
\hline Gender (male, female) & 1.746 & $0.842-6.561$ & 0.442 \\
\hline Ps $(0,1,2)$ & & & 0.020 \\
\hline 0 & 1 & I & \\
\hline I & 3.461 & $0.562-5.561$ & \\
\hline 2 & 2.651 & $0.162-7.381$ & \\
\hline Location of tumor (right, left, bilobar) & & & 0.712 \\
\hline Right lobe & 1 & I & \\
\hline Left lobe & 0.471 & $0.082-8.46 I$ & \\
\hline Bilobar & 1.291 & $0.232-5.762$ & \\
\hline Number of tumors $(\leq 3,>3)$ & 1.341 & $0.119-4.593$ & 1.021 \\
\hline Lengths of the tumors $(\leq 10 \mathrm{~cm},>10 \mathrm{~cm})$ & 1.797 & $0.852-3.794$ & 0.365 \\
\hline Metastasis & 1.934 & $0.361-4.812$ & 0.102 \\
\hline Child-Pugh (A, B) & 0.734 & $0.084-7.568$ & 0.341 \\
\hline CEA & 3.281 & $1.452-7.409$ & 0.477 \\
\hline CA199 & 1.571 & $0.458-6.451$ & 0.737 \\
\hline $\operatorname{AFP}(\leq 200 \mathrm{ng} / \mathrm{mL},>200 \mathrm{ng} / \mathrm{mL})$ & 0.973 & $0.584-1.62 \mid$ & 0.916 \\
\hline Deposition of lipiodol & & & 1.235 \\
\hline Mild & 1 & I & \\
\hline Moderate & 0.572 & $0.161-2.678$ & \\
\hline Good & 0.678 & $0.091-2.343$ & \\
\hline Tumor response (PD,SD,PR,CR) & & & 0.048 \\
\hline PD & 1 & I & \\
\hline SD & 0.783 & $0.303-2.123$ & \\
\hline PR & 1.237 & $0.462-1.46 \mid$ & \\
\hline CR & 0.982 & $0.562-1.732$ & \\
\hline Number of sessions of TACE & 0.650 & $0.274-1.129$ & 0.157 \\
\hline
\end{tabular}

Abbreviations: HR, hazard ratio; Cl, confidence interval; PS, performance status; CEA, carcinoembryonic antigen; CAI99, carbohydrate antigen I99; AFP, alpha-fetal protein; PD, alpha-fetal protein; SD, stable disease; PR, partial response; CR, complete response; TACE, transarterial chemoembolization.

cycle. If the treatment plan was terminated, all patients were assessed for their cumulative toxicities.

\section{Statistical Analysis}

For analyzing PFS and OS, all patients were followed up until disease progression, death or lost to follow-up. PFS and OS were calculated using Kaplan-Meier method. PFS was defined as the time between the first TACE procedure till disease progression, and OS was calculated from the date of the first TACE till death or last follow-up visit. Cox proportional-hazards regression model was used to assess the independent prognostic factors of OS. Statistical analysis was performed using SPSS version 16.0 software (SPSS Inc., Chicago, IL, USA).

\section{Results}

Between April 15, 2014, and November 25, 2015, a total of 123 patients were recruited into the study (16 patients from Shandong Medical Imaging Research Institute, 7 from Shandong Cancer Hospital and Institute, 29 from Qilu Hospital of Shandong University, 25 from Affiliated Hospital of Qingdao University, 28 from Qingdao Central Hospital, 11 from 960 Hospital of People's Liberation Army, 5 from Qianfoshan Hospital of Shandong Province and 2 from People's Hospital of Jining City). The detailed baseline demographics and disease characteristics of the patients are shown in Table 1.

A total of 452 cycles of TACE with raltitrexed plus oxaliplatin were performed. Three patients completed 1 
cycle of TACE, 120 patients completed at least 2 cycles of TACE (4 patients completed 2 cycles, 23 patients completed 3 cycles and 93 patients completed 4 cycles) and were evaluated for tumor response. Finally, 117 patients were followed up for PFS and OS.

\section{Tumor Response}

All 123 patients were evaluated for tumor response. Of these, 10 patients achieved a complete response (CR), 43 patients achieved a partial response (PR), 65 patients had stable disease (SD) and 5 patients had disease progression (PD). The objective response rate (ORR) was $43.09 \%$ and the disease control rate (DCR) was $95.5 \%$.

\section{PFS And OS}

A total of 117 patients were subjected to follow-up. The median duration of follow-up was 20 months (ranging from 1.9 to 41.5 months). Twenty-two patients were alive on June 30, 2018, and were censored at that time point. The median PFS was 338 days $(95 \%$ CI: 302, 704) (Figure 1). The median OS time was 623 days (95\% CI: 461, 785) (Figure 2).

The Cox proportional-hazards regression model indicated that age, ECOG performance status and response to TACE were independent prognostic factors of OS (Table 2). Patients who achieved CR or PR after two cycles of TACE showed better OS than patients who achieved CR or PR after 4 cycles of TACE or sustained stable disease (SD) (Figure 3).

\section{Safety}

No treatment-related mortality occurred within 30 days of treatment procedure. The most common complications included post-embolization syndrome, liver dysfunction and hematological toxicity. No grade 4 adverse events occurred. The incidence of toxicity is summarized in Table 3.

\section{Discussion}

$\mathrm{HCC}$ is the seventh most common cancer and third leading cause of cancer-related deaths worldwide. ${ }^{14}$ Most of the HCC patients are diagnosed in the late stage and are not candidates for curative options such as liver transplantation and surgical resection. TACE is recommended as the mainstay treatment for patients with unresectable HCC. Raltitrexed can be administered as a short-term infusion or mixed with lipiodol, and so it is regarded as a better candidate for TACE when compared with 5-fluorouracil. A retrospective study showed that raltitrexed plus oxaliplatin regimen of TACE had a longer PFS time than traditional 5-fluorouracil-based TACE and was safe

\section{Survival Functions}

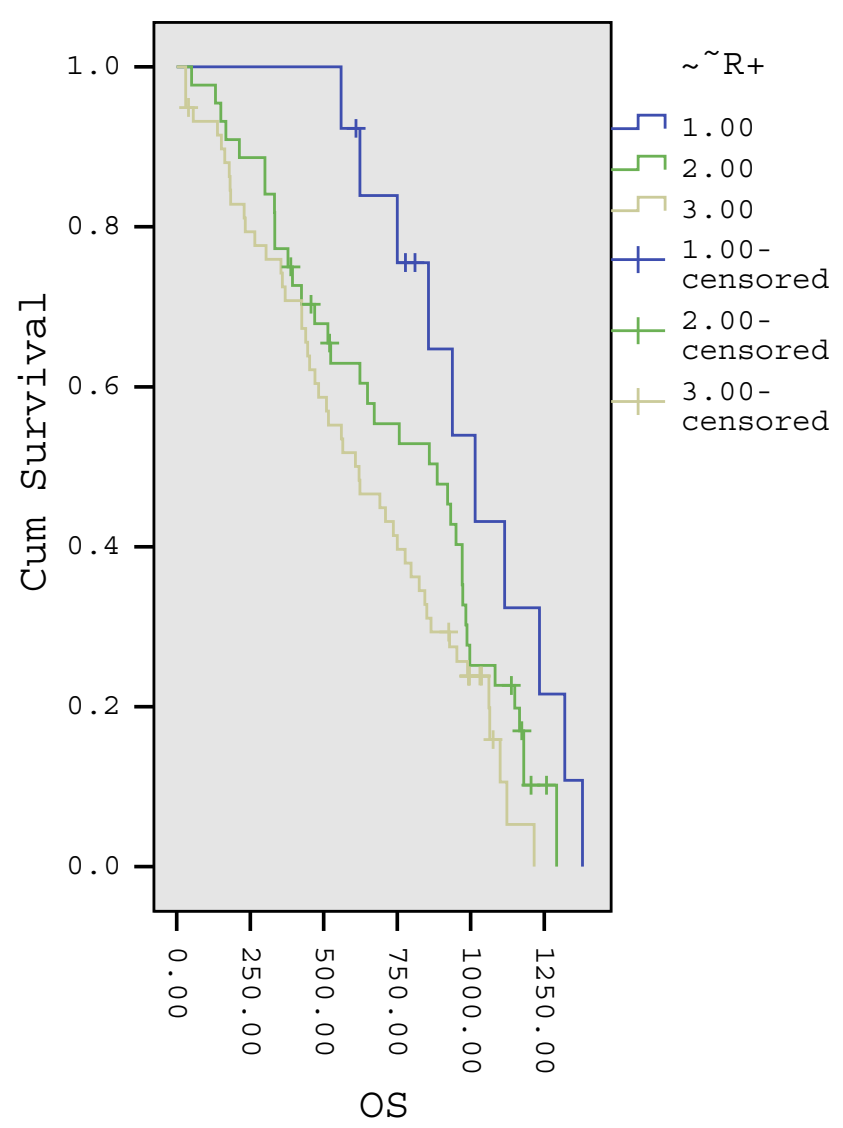

Figure 3 Kaplan - Meier curves generated for 117 patients to compare the median overall survival according to blue I, CR or PR after 2 cycles of TACE, green 2, CR or PR after 4 cycles of TACE and yellow 3 SD after 4 cycles of TACE.

and tolerable in patients with unresectable HCC. ${ }^{15}$ According to a prospective, multicenter, randomized study, raltitrexedbased TACE had a better disease control rate and the median OS and median PFS of raltitrexed-based TACE were significantly higher than those obtained by 5-fluorouracil-based TACE and doxorubicin-based TACE (OS: 13.4 vs. 9.6 vs. 8.5 months; PFS: 6.7 vs. 4.9 vs. 4.6 months). ${ }^{16}$ The disease control rate of our study was similar to that of the previous study, but the median PFS and OS of our study are longer than those obtained in the previous study. We presumed that this might be due to the completion of more cycles of TACE in our study than the previous study. Majority of patients (i.e., 94\%) in our study completed at least 3 cycles of TACE and threefourths $(75 \%)$ of patients completed 4 cycles of TACE when compared with those who completed 3 cycles of TACE (48\%) in the previous study. Repeated TACE procedures can prolong OS in patients with unresectable HCC. ${ }^{17,18}$ For patients who had PR or SD after a session of TACE, repeated TACE might 
Table 3 Common Treatment-Related Adverse Events Occurred Among Entire Population

\begin{tabular}{|l|l|l|l|l|l|l|}
\hline & AE & I n (\%) & II n (\%) & III n (\%) & IV n (\%) & Total n (\%) \\
\hline Hematological toxicity & RBC & $32(26.0 \%)$ & $3(2.4 \%)$ & $0(0.0 \%)$ & $0(0.0 \%)$ & $35(28.5 \%)$ \\
& WBC & $29(23.6 \%)$ & $5(4.1 \%)$ & I (0.8\%) & $0(0.0 \%)$ & $35(28.5 \%)$ \\
& HB & $36(29.3 \%)$ & $5(4.1 \%)$ & $0(0.0 \%)$ & $0(0.0 \%)$ & $4 I(33.3 \%)$ \\
& NEU & $18(14.6 \%)$ & $4(3.3 \%)$ & $0(0.0 \%)$ & $0(0.0 \%)$ & $22(17.9 \%)$ \\
& PLT & $34(27.6 \%)$ & $6(4.9 \%)$ & $2(1.6 \%)$ & $0(0.0 \%)$ & $42(34.1 \%)$ \\
\hline \multirow{2}{*}{ Liver dysfunction } & TBIL & $28(22.8 \%)$ & $1(0.8 \%)$ & $0(0.0 \%)$ & $0(0.0 \%)$ & $29(23.6 \%)$ \\
& DBIL & $31(25.2 \%)$ & $1(0.8 \%)$ & $0(0.0 \%)$ & $0(0.0 \%)$ & $32(26.0 \%)$ \\
& ALB & II (8.9\%) & $0(0.0 \%)$ & $0(0.0 \%)$ & $0(0.0 \%)$ & $11(8.9 \%)$ \\
& ALP & $18(14.6 \%)$ & $8(6.5 \%)$ & $7(5.7 \%)$ & $0(0.0 \%)$ & $33(26.8 \%)$ \\
& BUN & $9(7.3 \%)$ & $0(0.0 \%)$ & $0(0.0 \%)$ & $0(0.0 \%)$ & $9(7.3 \%)$ \\
& Cr & $19(15.4 \%)$ & $0(0.0 \%)$ & $0(0.0 \%)$ & $0(0.0 \%)$ & $19(15.4 \%)$ \\
& ALT & $34(27.6 \%)$ & $12(9.8 \%)$ & $5(4.1 \%)$ & $0(0.0 \%)$ & $5 I(41.5 \%)$ \\
& AST & $39(31.7 \%)$ & $8(6.5 \%)$ & $3(2.4 \%)$ & $0(0.0 \%)$ & $50(40.7 \%)$ \\
\hline \multirow{2}{*}{ Postembolization syndrome } & Fever & $95(77.2 \%)$ & $13(10.6 \%)$ & $0(0.0 \%)$ & $0(0.0 \%)$ & $108(87.8 \%)$ \\
& Abdominal pain & $8 I(65.9 \%)$ & $8(6.5 \%)$ & $16(13.0 \%)$ & $0(0.0 \%)$ & $105(85.4 \%)$ \\
& Nausea and vomiting & $43(35.0 \%)$ & $16(13.0 \%)$ & $0(0.0 \%)$ & $0(0.0 \%)$ & $59(48.0 \%)$ \\
\hline
\end{tabular}

Abbreviations: RBC, red blood cell; WBC, white blood cell; HB, hemoglobin; NEU, neutrophil; PLT, platelet; TBIL, total bilirubin; DBIL, direct bilirubin; ALB, albumin; ALP, alkaline phosphatase; BUN, blood urea nitrogen; $\mathrm{Cr}$, creatinine; ALT, alanine transaminase; AST, aspartate transaminase.

give patients a chance to achieve $\mathrm{CR}$ or $\mathrm{PR}$, or prevent rapid disease progression, prolonging the PFS. ${ }^{19}$ However, Cox proportional-hazards regression model in the current study indicated that the number of TACE sessions was not an independent prognostic factor, while age, ECOG performance status and response to TACE were independent prognostic factors of OS. Sub-group analysis showed that patients who achieved CR or PR after 2 cycles of TACE had better OS than those who achieved CR or PR after 4 cycles of TACE and patients who sustained SD. The results of the current study achieved a tumor response rate at an early time point, predicting good survival benefit in patients with HCC undergoing TACE. This was in concordance with the results of the previous study. ${ }^{20}$

Our study also evaluated the safety and toxicity of raltitrexed plus oxaliplatin-based TACE in patients with unresectable HCC. No treatment-related mortalities occurred within 30 days of treatment procedure. The most common complications were similar with that of the traditional 5fluorouracil and doxorubicin-based TACE. No grade 4 adverse events were observed. The results indicated that raltitrexed plus oxaliplatin-based TACE is safe and tolerable in patients with unresectable HCC.

\section{Conclusion}

Our study showed that raltitrexed plus oxaliplatin-based TACE led to high tumor response rate and promising PFS and OS, and was safe and tolerable in patients with unresectable HCC. This regimen might be a good choice for unresectable HCC patients treated with TACE.

\section{Disclosure}

The authors report no conflicts of interest in this work.

\section{References}

1. Cheung TT, Kwok PC, Chan S, et al. Hong Kong consensus statements for the management of unresectable hepatocellular carcinoma. Liver Cancer. 2018;7:40-54. doi:10.1159/000485984

2. Omata M, Cheng AL, Kokudo N, et al. Asia-Pacific clinical practice guidelines on the management of hepatocellular carcinoma: a 2017 update. Hepatol Int. 2017;11:317-370. doi:10.1007/s12072-017-97999

3. Bruix J, Sherman M. Management of hepatocellular carcinoma: an update. Hepatology. 2011;53:1020-1022. doi:10.1002/hep.24199

4. Liapi E, Geschwind JF. Intra-arterial therapies for hepatocellular carcinoma: where do we stand? Ann Surg Oncol. 2010;17:1234-1246. doi:10.1245/s10434-010-0977-4

5. Qin S, Bai Y, Lim HY, et al. Randomized, multicenter, open-label study of oxaliplatin plus fluorouracil/leucovorin versus doxorubicin as palliative chemotherapy in patients with advanced hepatocellular carcinoma from Asia. J Clin Oncol. 2013;31:3501-3508. doi:10.12 00/JCO.2012.44.5643

6. Kemeny NE, Niedzwiecki D, Hollis DR, et al. Hepatic arterial infusion versus systemic therapy for hepatic metastases from colorectal cancer: a randomized trial of efficacy, quality of life, and molecular markers (CALGB 9481). J Clin Oncol. 2006;24:1395-1403. doi:10.1200/JCO. 2005.03.8166

7. Mocellin S, Pilati P, Lise M, Nitti D. Meta-analysis of hepatic arterial infusion for unresectable liver metastases from colorectal cancer: the end of an era? J Clin Oncol. 2007;25:5649-5654. doi:10.1200/JCO. 2007.12.1764 
8. Mocellin S, Pasquali S, Nitti D. Fluoropyrimidine-HAI (hepatic arterial infusion) versus systemic chemotherapy (SCT) for unresectable liver metastases from colorectal cancer. Cochrane Database Syst Rev. 2009;3:CD007823. doi:10.1002/14651858.CD007823.pub2

9. Gravalos C, Salut A, Garcia-Giron C, et al. A randomized phase II study to compare oxaliplatin plus 5-fluorouracil and leucovorin (FOLFOX4) versus oxaliplatin plus raltitrexed (TOMOX) as firstline chemotherapy for advanced colorectal cancer. Clin Transl Oncol. 2012;14:606-612. doi:10.1007/s12094-012-0843-X

10. Liu Y, Wu W, Hong W, et al. Raltitrexed-based chemotherapy for advanced colorectal cancer. Clin Res Hepatol Gastroenterol. 2014;38:219-225. doi:10.1016/j.clinre.2013.11.006

11. Khouri C, Guiu B, Cercueil JP, et al. Raltitrexed and oxaliplatin hepatic arterial infusion for advanced colorectal cancer: a retrospective study. Anticancer Drugs. 2010;21:656-661. doi:10.1097/CAD.0b013e32833 $7 \mathrm{~d} 469$

12. Guo JH, Zhang HY, Gao S, et al. Hepatic artery infusion with raltitrexed or 5-fluorouracil for colorectal cancer liver metastasis. World $J$ Gastroenterol. 2017;23:1406-1411. doi:10.3748/wjg.v23.i8.1406

13. Shao G, Liu R, Ding W, et al. Efficacy and safety of raltitrexed-based transarterial chemoembolization for colorectal cancer liver metastases. Anticancer Drugs. 2018;29:1021-1025. doi:10.1097/CAD.0000000000 000690

14. Kudo M. The 2008 Okuda lecture: management of hepatocellular carcinoma: from surveillance to molecular targeted therapy. $J$ Gastroenterol Hepatol. 2010;25:439-452. doi:10.1111/j.1440-1746. 2009.06207.x
15. Cui W, Fan W, Zhang Q, et al. Comparison of two transarterial chemoembolization regimens in patients with unresectable hepatocellular carcinoma: raltitrexed plus oxaliplatin versus 5-fluorouracil plus oxaliplatin. Oncotarget. 2017;45:79165-79174. doi:10.18632/ ontogarget.16298

16. Zhao C, Fan L, Qi F, et al. Raltitrexed plus oxaliplatin-based transarterial chemoembolization in patients with unresectable hepatocellular carcinoma. Anticancer Drugs. 2016;27:689-694. doi:10.1097/ CAD.0000000000000371

17. Kim DY, Ryu HJ, Choi JY, et al. Radiological response predicts survival following transarterial chemoembolisation in patients with unresectable hepatocellular carcinoma. Aliment Pharmacol Ther. 2012;35:1343-1350. doi:10.1111/j.1365-2036.2012.05089.x

18. Georgiades C, Geschwind JF, Harrison N, et al. Lack of response after initial chemoembolization for hepatocellular carcinoma: does it predict failure of subsequent treatment? Radiology. 2012;265:115123. doi:10.1148/radiol.12112264

19. Shim JH, Lee HC, Kim SO, et al. Which response criteria best help predict survival of patients with hepatocellular carcinoma following chemoembolization? A validation study of old and new models. Radiology. 2012;262:708-718. doi:10.1148/radiol.11110282

20. Kim BK, Kim SU, Kim KA, et al. Complete response at first chemoembolization is still the most robust predictor for favorable outcome in hepatocellular carcinoma. J Hepatol. 2015;62:1304-1310. doi:10.1016/j.jhep.2015.01.022

\section{Publish your work in this journal}

Cancer Management and Research is an international, peer-reviewed open access journal focusing on cancer research and the optimal use of preventative and integrated treatment interventions to achieve improved outcomes, enhanced survival and quality of life for the cancer patient.
The manuscript management system is completely online and includes a very quick and fair peer-review system, which is all easy to use Visit http://www.dovepress.com/testimonials.php to read real quotes from published authors. 